\title{
Association of Clinical Parameters and Prognosis with the Pretreatment Systemic Immune-inflammation Index (SII) in Patients with Gastric Cancer
}

\author{
Xihuang $\mathrm{Cao}^{1}$, Jiaming Xue ${ }^{2}$, Huiliang Yang ${ }^{2}$, Xiao $\mathrm{Han}^{2}$ and Guo Zu \\ ${ }^{1}$ Department of Emergency Surgery, The Dalian Municipal Central Hospital, China \\ ${ }^{2}$ Department of General Surgery, The Dalian Municipal Central Hospital, China
}

\begin{abstract}
This study explored the relationship between the pretreatment systemic immune-inflammation index (SII) and overall survival (OS) in gastric cancer (GC) patients. A systemic literature search was performed to find out the articles that estimated the relationship of SII with specific clinical parameters and OS in GC patients. Nine articles (including 10 studies) were included. A total of 3,850 cases were eventually included. In GC patients, there was no association between pretreatment SII and gender $(\mathrm{OR}=0.991, \mathrm{p}=0.944)$ or differentiation $(\mathrm{OR}=1.093, \mathrm{p}=0.687)$. However, pretreatment SIl was related to depth of tumor invasion $(O R=0.340, p<0.001)$, lymph node metastasis $(O R=0.447, p<0.001)$ and TNM stage $(O R=0.361, p<0.001)$ in $G C$ patients. The ORs of 1-year, 3-year and 5-year OS were $0.467\left(I^{2}=0.0 \% ; p=0.682\right), 0.355\left(I^{2}=85.6 \% ; p<0.001\right)$ and $0.507\left(I^{2}=56.4 \%\right.$; $p=0.057$ ). The pretreatment SII could be used as an indicator of the depth of tumor invasion, lymph node metastasis, TNM stage and overall of gastric cancer patients. However, more multi-centres researches are needed to confirm these findings.
\end{abstract}

Key Words: Systemic immune-inflammation index (SII), Prognosis, Gastric cancer.

How to cite this article: Cao X, Xue J, Yang H, Han X, Zu G. Association of Clinical Parameters and Prognosis with the Pretreatment Systemic Immune-inflammation Index (SII) in Patients with Gastric Cancer. J Coll Physicians Surg Pak 2021; 31(01):83-88.

\section{INTRODUCTION}

As one of the most common digestive tract cancers, gastric cancer (GC) is still one of the leading causes of cancer-associated mortality worldwide. ${ }^{1}$ Although prognosis of GC patients has improved during the past decades, radical gastrectomy is still the most important treatment for GC, and the survival rate is still much less than $30 \% .{ }^{2}$ Recurrence and metastasis occur in $35-70 \%$ of GC patients within 5 years and are the most important poor prognostic factors, even after radical resection. ${ }^{3}$ Therefore, there is a strong need to find out new markers and to predict the overall survival (OS) of GC patients.

Several studies have shown that inflammatory factors and cells are involved in the tumor microenvironment. ${ }^{4,5}$ In recent years, clinical studies have confirmed that the immune and inflammatory cells play important roles in the progression, invasion and metastasis of several tumors.$^{6-8}$ Some inflammatory biomarkers, such as the lymphocyte-to-monocyte ratio (LMR), are used to predict the OS, tumor recurrence and metastasis of cancers, including GC. ${ }^{9,10}$

Correspondence to: Dr. Guo Zu, Department of General Surgery, The Dalian Municipal Central Hospital, China E-mail: zushanghai@163.com

Received: March 19, 2020; Revised: August 02, 2020;

Accepted: August 24, 2020

DOI: https://doi.org/10.29271/jcpsp.2021.01.83
Recently, a novel index named SII (SII = neutrophil $\times$ platelet $/$ lymphocyte), which is based on systemic immune-inflammation index, has become a better marker to reflect the inflammation and immune status of host; it has been used as a prognostic index in bladder cancer, colorectal cancer and lung cancer. ${ }^{11-13}$ However, it has not reached a consensus between the pretreatment SII and OS of several tumor patients, including GC patients. Chen et al. reported that a low pretreatment SII was significantly related to gender, lymph node stage, and tumor size. ${ }^{14}$ Wang et al. reported that pretreatment SII was related to age, Borrmann type, lymph node and distant metastasis, high CEA levels, TNM stage, tumor size, and tumor invasion. ${ }^{15}$ Therefore, the presentmeta-analysis was performed to check the relationship of clinical parameters and prognostic values of GC patients to the pretreatment SII.

\section{METHODOLOGY}

The studies about pretreatment SII and GC were searched in the databases of Cochrane Library, PubMed, Springer, EMBASE, Elesevier, Web of Science, and Chinese databases (including CNKI, Wanfang and VIP). The time of literature retrieval was until Dec 15, 2019. The key words were used as stomach, gastric, tumor, cancer, carcinoma, neoplasm and systemic immune-inflammation index or SII.

The inclusion criteria of the relevant studies were: articles including data on the pretreatment SII, clinical parameters and OS of GC patients; the data of neutrophil, platelet and lympho- 
cyte must be included and measured before treatment, such as chemoradiotherapy, surgery or targeted therapy; and the articles were estimated by Newcastle-Ottawa Quality Assessment Scale (NOS) score $>6$. The exclusion criteria for the article were: lack of data on clinical parameters or OS and articles; duplicated data; patients with infection, hematological or autoimmune diseases; and those patients who took medicine that could influence the neutrophil, platelet and lymphocyte counts.

The included articles in this study were independently completed by two authors (Xihuang Cao and Jiaming Xue). Two authors (Xihuang Cao and Jiaming Xue) extracted the needed data independently. Any disagreement was resolved by another author (Huiliang Yang). The following information extracted from the articles included: name of authors, journal, published year, country of the patients, gender, number of cases, depth of tumor invasion, TNM stage, lymph node metastasis, methods of treatment, period of follow-up, cut-off value of pretreatment SII, and OS of GC patents.

The quality evaluation of the eligible articles was independently done by two researchers (Xihuang Cao and Jiaming Xue) according to the NOS. Study with a score $\geq 6$ was included in this study.

STATA 10.0 (Stata Corporation) software was used in this analysis. The relationship between pretreatment SII and OS of GC patients were evaluated by odds ratios (ORs) and $95 \%$ confidence intervals ( $\mathrm{Cls}$ ) with the fixed effects model. The heterogeneity analysis was checked by Higgins $I^{2}$ and Cochran's Q test. $I^{2}>50 \%$ and/or $p<0.10$ were defined as significant heterogeneity. If $\mathrm{I}^{2}>50 \%$ and/or $p<0.10$, the random effects model was used.

We used the Begg'stest and Egger's test to estimate the publication bias, and $p<0.05$ was considered to significant statistical differences.

\section{RESULTS}

The literature review and flow diagram of this analysis are shown in Figure 1. Twenty-nine articles were retrieved from the database. After eliminating two duplicate studies and other 18 articles, including lack of clinical data and review, nine studies were included by reading the full-texts. One article included two validation cohorts; so finally, nine articles (including 10 studies) including 3,850 patients were included. ${ }^{14-22}$

The eligible 10 studies were published from 2015 to 2019. The included number of patients varied between 60 and 1,032. The studies were conducted in Korea (one study) and China (nine studies). ${ }^{14-22}$ The cut-off values of pretreatment SII were checked from 320 to 888 . The characteristics and results of the eligiblestudies were listed in Tablel.

To analyse the relationship between pretreatment SII and clinical parameters and OS of GC patients, the correlation of pretreatment SII and parameters were assessed in more than three studies. As shown in Table II and Figure 2, there was no association between pretreatment SII and gender $(\mathrm{OR}=0.991$, $p=0.944)$ or differentiation $(\mathrm{OR}=1.093, p=0.687)$ in $\mathrm{GC}$ patients. However, pretreatment SII was related to depth of tumor invasion $(\mathrm{OR}=0.340, p<0.001)$, lymph node metastasis $(\mathrm{OR}=0.447, p<0.001)$ and TNM stage $(\mathrm{OR}=0.361, p<0.001)$.

As shown in Figure 3, the Begg's funnel plot was symmetric, and the Egger's test of gender, differentiation, T, N and TNM also showed $p$ values of $0.599,0.116,0.112,0.796$ and 0.293 , respectively. The publication bias was not significant.

Seven studies have estimated the relationship between pretreatment SII and the 1-year, 3-year and 5-year OS of GC patients. The results of eligible studies are shown in Table II and III. Patients with high pretreatment SII have higher relative risks of poor OS than patients with low pretreatment SII. The 1-year, 3-year and 5-year patients OS had OR of $0.467\left(I^{2}=0.0 \% ; p=0.682\right), 0.355$ $\left(I^{2}=85.6 \% ; p<0.001\right)$ and $0.507\left(I^{2}=56.4 \% ; p=0.057\right.$, Figure 4$)$.

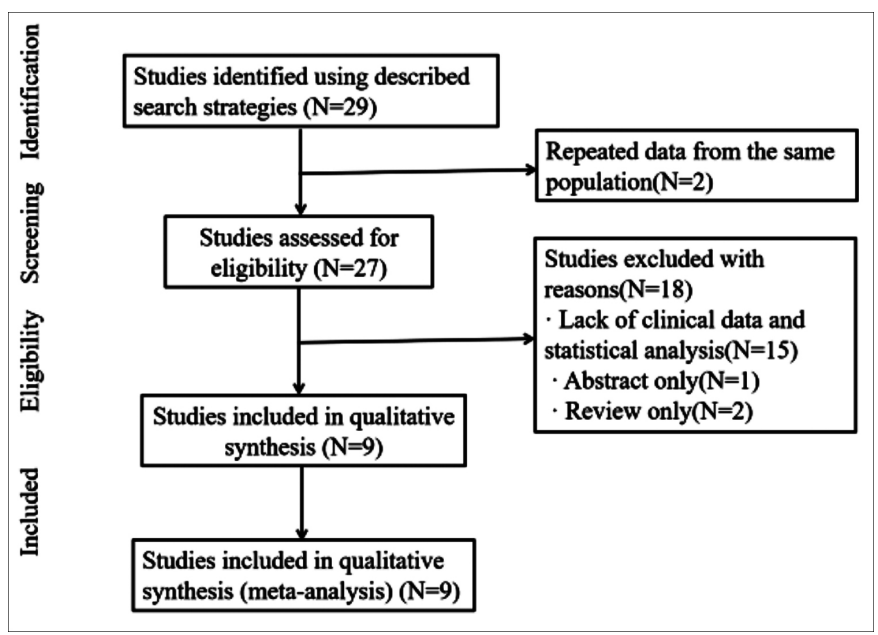

Figure 1: Flow diagram of the literature review.

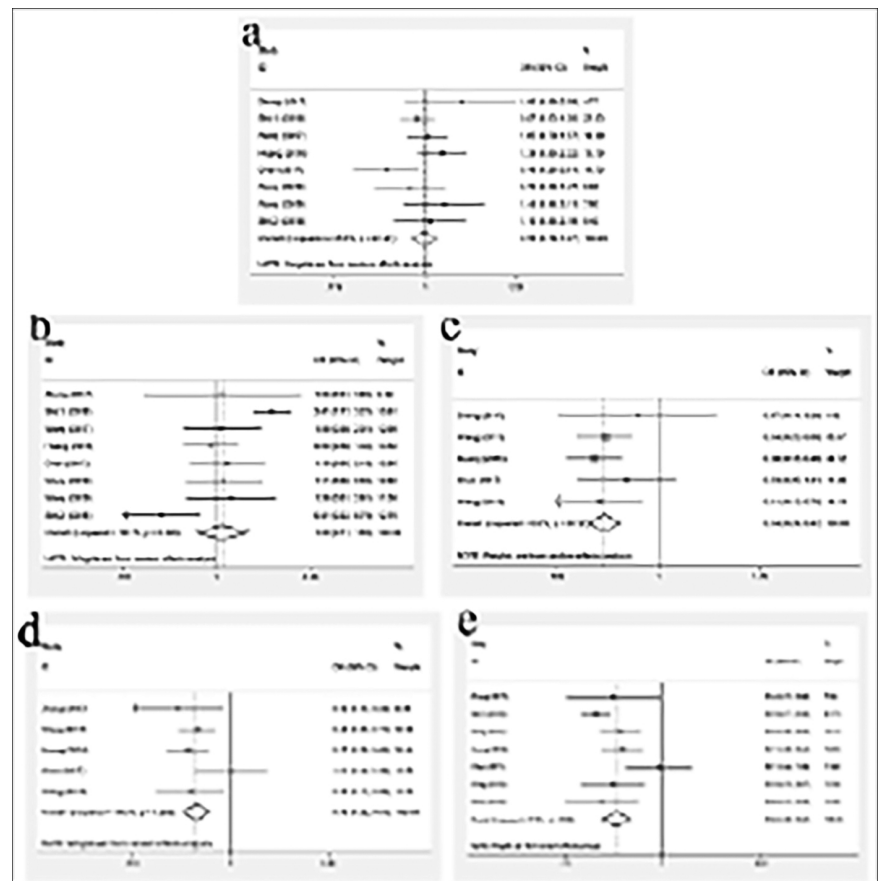

Figure 2: Forest plots showing the correlation between pretreatment SII and gender (a), differentiation (b), depth of invasion (c), lymph node metastasis (d), and TNM stage (e). 


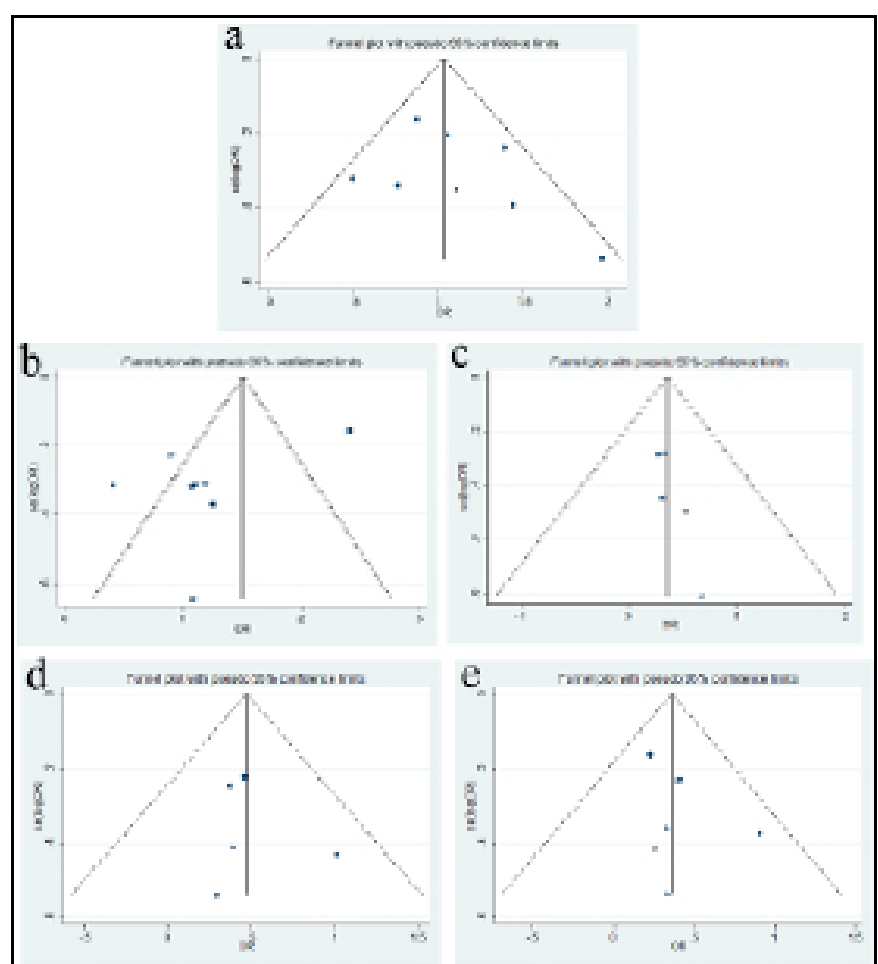

Figure 3: Egger's funnel plotestimated the publication bias of the correlation between pretreatment SII and gender (a), differentiation (b), depth of tumorinvasion (c), lymph node metastasis (d), and TNM stage (e).

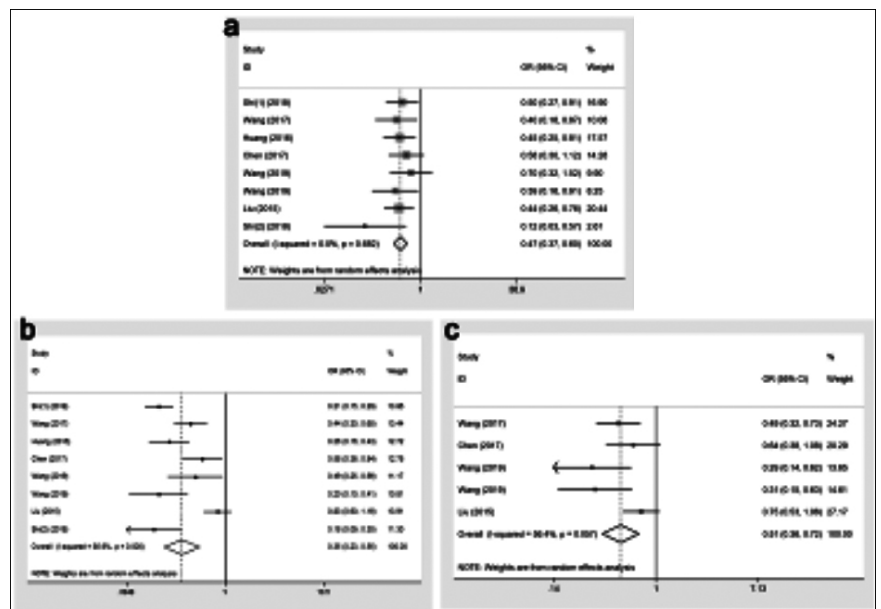

Figure 4: Forest plots showing the correlation between pretreatment SII and 1-yearOS (a), 3-yearOS (b), and 5-yearOS (c).

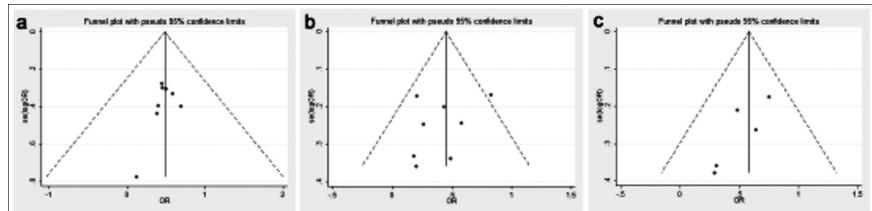

Figure 5: Egger's funnel plotestimated the publication bias of the correlation between pretreatment SII and 1-year OS (a), 3-year OS (b), and 5yearOS (c).

As shown in Figure 5, Begg's funnel plots of OS (1-year, 3-year and 5-year) were symmetric, and the Egger's testfor the 1-year, 3-year and 5-year OS indicated no significant publication bias ( $p=0.102,0.459$ and 0.063 , respectively).

\section{DISCUSSION}

It has been reported that cancer-related immunity and inflammation are essential components of the tumor microenvironment which is related to tumor development. Immunity and inflammation is associated with tumor progression. ${ }^{23,24} \mathrm{As}$ an immune and inflammation-related biomarker, pretreatment high SII is a poor prognostic factor of several tumors, including GC. In this study, the association between preoperative SII and clinical parameters, and OS of GC patients were confirmed. In GC patients, it was found that high pretreatment SII was related to depth of invasion, lymph node metastasis and TNM stage; and a high pretreatment SII was associated with poorer OS of GC patients.

In GC, many immune and inflammatory cells compose the tumor stroma and microenvironment. ${ }^{25,26}$ They can also secrete cytokines and inflammatory factors, which may contribute to the recurrence and metastasis of tumor. ${ }^{27}$ For example, CXCR1/CXCR2 and interleukin- 6 are involved in the processes of tumor cell proliferation, invasion and metastasis. ${ }^{28,29}$ As an inflammation-related biomarker, pretreatment SII has been shown as an important indicator in several cancers. ${ }^{11-13}$ It has been reported that SII is associated with clinical parameters in $\mathrm{GC}$, but there is still controversy. In this meta-analysis, high pretreatment SII was correlated with depth of invasion, lymph node metastasis, and TNM stage. However, there wasno association between pretreatment SII and gender $(p=0.944)$ or differentiation ( $p=0.687$ ) in GC patients.

Several studies have investigated the association between the pretreatment SII and the OS of malignant tumors patients, and the high pretreatment SII predicted prognostic value in many types of tumors. High pretreatment SII is related to poor OS in several kinds of malignant cancer patients. ${ }^{30-32}$ The pretreatment SII is an immune and inflammation-related index and has been used to reflect inflammation and immune status. The tumor-associated neutrophils are involved in progression of tumor. ${ }^{33}$ The platelet count and mean platelet volume is related to the regional details of the microenvironment of pancreatic neuroendocrine tumor. ${ }^{34}$ Moreover, lymphocytes are involved in infiltrating the tumor environment. ${ }^{35}$ In this study, a meta-analysis was conducted to detect the association of pretreatment SII and OS in GC patients. The results showed that high pretreatment SII had poorer OS than those with low pretreatment SII in GC patients.

This meta-analysis also has several limitations. First, only 10 studies were from China or Korea, which involved 3,850 patients, all from East Asia, and the results from other countries or regions remain unclear. So the bias cannot be ignored.

Second, the data was just from the published paper, and lacked the original data for some parameters. The subgroup analysis could not be performed, which may also cause bias. 
Table I: Main characteristics and results of the eligible studies.

\begin{tabular}{|c|c|c|c|c|c|c|c|c|c|}
\hline No. & First author & Year & Case No. & M/F & During & Country & $\begin{array}{l}\text { Cutoff } \\
\text { value }\end{array}$ & Treatment & NOS \\
\hline 1 & Zheng $^{16}$ & 2017 & 60 & $36 / 24$ & $2015.4-2016.3$ & China & 888 & Surgery & 9 \\
\hline 2 & Shi $(1)^{17}$ & 2018 & 688 & $471 / 217$ & 2012-2014 & China & 320 & Surgery & 7 \\
\hline 3 & $\operatorname{Shi}(2)^{17}$ & 2018 & 174 & $131 / 43$ & 2012-2014 & China & 320 & Surgery & 8 \\
\hline 4 & Guner $^{18}$ & 2018 & 1032 & $667 / 365$ & 2009.3-2015.12 & Korea & - & Surgery & 9 \\
\hline 5 & Wang $^{19}$ & 2017 & 444 & $281 / 163$ & 1994.1-2005.12 & China & 660 & Surgery & 6 \\
\hline 6 & Huang $^{20}$ & 2016 & 455 & $305 / 150$ & 2013.1-2014.12 & China & 572 & Surgery & 6 \\
\hline 7 & Chen $^{14}$ & 2017 & 185 & $125 / 60$ & 2007.7-2015.9 & China & 600 & Surgery & 7 \\
\hline 8 & Wang $^{15}$ & 2019 & 182 & $133 / 49$ & 2009.1-2012.12 & China & 600 & Surgery & 7 \\
\hline 9 & Wang $^{21}$ & 2019 & 175 & $127 / 48$ & $2008.1-2015.8$ & China & 782 & Surgery & 6 \\
\hline 10 & $\mathrm{Liu}^{22}$ & 2015 & 455 & $314 / 141$ & $2005.1-2010.12$ & China & 660 & Surgery & 6 \\
\hline
\end{tabular}

Table II: Results of clinical parameters and prognostic value of SII in patients with GC.
\begin{tabular}{|l|c|c|c|}
\hline Clinical parameters & No. of studies & Overall OR (95\%CI) & Heterogeneity test (Q, I', P) \\
\hline Gender & $1,2,3,5,6,7,8,9$ & $0.991(0.776-1.266)$ & $10.82,35.3 \%, 0.944($ random) \\
\hline Differentiation & $1,2,3,5,6,7,8,9$ & $1.093(0.708-1.688)$ & $32.03,78.1 \%, 0.687($ random) \\
\hline T & $1,5,6,7,9$ & $0.340(0.244-0.474)$ & $2.05,0.0 \%,<0.001($ random) \\
\hline N & $1,5,6,7,9$ & $0.447(0.325-0.614)$ & $4.95,19.2 \%,<0.001($ random) \\
\hline TNM & $1,2,3,5,6,7,9$ & $0.361(0.259-0.504)$ & $14.02,57.2 \%,<0.001($ random) \\
\hline 1-year OS & $2,3,5,6,7,8,9,10$ & $0.467(0.365-0.597)$ & $4.82,0.0 \%,<0.001($ random) \\
\hline 3-year OS & $2,3,5,6,7,8,9,10$ & $0.355(0.230-0.548)$ & $48.51,85.6 \%,<0.001($ random) \\
\hline 5-year OS & $5,7,8,9,10$ & $0.507(0.359-0.716)$ & $9.18,56.4 \%,<0.001($ random) \\
\hline
\end{tabular}

Table III: Prognostic values of the SII in GC patients.

\begin{tabular}{|l|l|l|l|l|l|l|}
\hline Author (year) & 1-Year & 3-Year & 5-Year \\
\hline & OR (95\%CI) & Weight & OR (95\%CI) & Weight & OR (95\%CI) \\
\hline Shi (1) (2018) & $0.499(0.275-0.908)$ & $16.90 \%$ & $0.207(0.148-0.289)$ & $13.86 \%$ & - & Weight \\
\hline Wang (2017) & $0.401(0.185-0.871)$ & $10.06 \%$ & $0.439(0.295-0.651)$ & $13.44 \%$ & $0.485(0.321-0.733)$ & - \\
\hline Huang (2016) & $0.452(0.251-0.812)$ & $17.57 \%$ & $0.263(0.162-0.428)$ & $12.72 \%$ & - & \\
\hline Chen (2017) & $0.584(0.300-1.119)$ & $14.28 \%$ & $0.584(0.362-0.942)$ & $12.78 \%$ & $0.645(0.385-1.081)$ & $20.29 \%$ \\
\hline Wang (2019) & $0.696(0.319-1.519)$ & $9.90 \%$ & $0.494(0.254-0.960)$ & $11.17 \%$ & $0.295(0.140-0.620)$ & $13.65 \%$ \\
\hline Wang (2019) & $0.385(0.164-0.907)$ & $8.25 \%$ & $0.204(0.101-0.414)$ & $10.81 \%$ & $0.310(0.153-0.626)$ & $14.61 \%$ \\
\hline Liu (2015) & $0.443(0.257-0.763)$ & $20.44 \%$ & $0.832(0.598-1.157)$ & $13.91 \%$ & $0.752(0.534-1.060)$ & $27.17 \%$ \\
\hline Shi (2) (2018) & $0.124(0.027-0.568)$ & $2.61 \%$ & $0.182(0.095-0.348)$ & $11.30 \%$ & - & - \\
\hline Overall & $0.467(0.365-0.597)$ & $100 \%$ & $0.355(0.230-0.548)$ & $100 \%$ & $0.507(0.359-0.716)$ & $100 \%$ \\
\hline
\end{tabular}

\section{CONCLUSION}

A high pretreatment SII was related to the depth of invasion, lymph node metastasis and TNM stage of GC patients. High pretreatment SII in GC patients indicated poor OS. The pretreatment SII could be used as a valuable index to predict the prognosis of GC patients. However, more research is still needed to further verify the findings of this meta-analysis.

\section{CONFLICT OF INTEREST:}

The authors declared no conflict of interest.

\section{ETHICAL APPROVAL:}

This article does not contain any studies with human participants or animals performed by any of the authors.

\section{PATIENTS' CONSENT:}

Informed consents were obtained from all individual participants included in the study.

\section{AUTHORS' CONTRIBUTION:}

GZ: Design the study; assisted in interpreting the results; assessed the data quality.
XC, JX: Did the literature search, screening and data extraction; drafted the manuscript.

XC, HY, XH: Performed critical appraisal of all included studies.

$X C$ : Analysed data and assisted in the writing of paper.

All authors read and approved the final manuscript.

\section{REFERENCES}

1. Thrift AP, El-Serag HB. Burden of gastric cancer. Clin Gastroenterol Hepatol 2020; 18(3):534-42. doi.org/ 10.1016/j.cgh.2019.07.045

2. Hashim D, Boffetta P, La Vecchia C, Rota M, Bertuccio P, Malvezzi $M$, et al. The global decrease in cancer mortality: Trends and disparities. Ann Oncol 2016; 27(5):926-33. doi: 10.1093/annonc/mdw027.

3. Wang Z, Zhang X, Hu J, Zeng W, Zhou Z. Clinico-pathological features and outcomes in patients undergoing radical resection for early gastric cancer with signet ring cell histology. J Visc Surg 2015; 152(6):357-61. doi: 10.1016/ j.jviscsurg.2015.09.021.

4. DiDonato JA, Mercurio F, Karin M. NF-KB and the link between inflammation and cancer. Immunol Rev 2012; 
246(1): 379-400. doi: 10.1111/j.1600-065X.2012.01099.x.

5. Thorsson V, Gibbs DL, Brown SD, Wolf D, Bortone DS, Ou Yang $\mathrm{TH}$, et al. The Immune Landscape of Cancer. Immunity 2018; 48(4): 812-30. doi.org/10.1016/j.immuni.2018. 03.023 .

6. Yu LX, Ling Y, Wang HY. Role of nonresolving inflammation in hepatocellular carcinoma development and progression. NPJ Precis Oncol 2018; 2(1): 6. doi: 10.1038/s41698018-0048-z.

7. Orozco-Morales M, Soca-Chafre G, Barrios-Bernal P, Hernández-Pedro N, Arrieta O. Interplay between cellular and molecular inflammatory mediators in lung cancer. Mediators Inflamm 2016; 2016:3494608. doi: 10.1155/ 2016/3494608.

8. Grizzi F, Bianchi P, Malesci A, Laghi L. Prognostic value of innate and adaptive immunity in colorectal cancer. World J Gastroenterol 2013; 19(2):174-84. doi: 10.3748/wjg. v19.i2.174.

9. Gaitanidis A, Wiseman D, El Lakis M, Nilubol N, Kebebew E, Patel D. Preoperative systemic inflammatory markers are prognostic indicators in recurrent adrenocortical carcinoma. J Surg Oncol 2019; 120(8):1450-5. doi: 10.1002/jso.25760.

10. Itoh S, Yugawa K, Shimokawa M, Yoshiya S, Mano Y, Takeishi K, et al. Prognostic significance of inflammatory biomarkers in hepatocellular carcinoma following hepatic resection. BJS Open 2019; 3(4):500-8. doi: 10.1002/ bjs5.50170.

11. Berardi R, Santoni M, Rinaldi S, Bower M, Tiberi M, Morgese $F$, et al. Pre-treatment systemic immune-inflammation represents a prognostic factor in patients with advanced non-small cell lung cancer. Ann Transl Med 2019; 7(20):572. doi: 10.21037/atm.2019.09.18.

12. Zhang W, Wang R, Ma W, Wu Y, Maskey N, Guo Y, et al. Systemic immune-inflammation index predicts prognosis of bladder cancer patients after radical cystectomy. Ann Transl Med 2019; 7(18):431. doi: 10.21037/atm. 2019.09.02.

13. Lu Y, Xin D, Wang F. Predictive significance of preoperative systemic immune-inflammation index determination in postoperative liver metastasis of colorectal cancer. Onco Targets Ther 2019; 12:7791-9. doi: 10.2147/OTT.S223419.

14. Chen L, Yan Y, Zhu L, Cong X, Li S, Song S, et al. Systemic immune-inflammation index as a useful prognostic indicator predicts survival in patients with advanced gastric cancer treated with neoadjuvant chemotherapy. Cancer Manag Res 2017; 9: 849-67. doi: 10.2147/CMAR.S151026.

15. Wang Q, Zhu D. The prognostic value of systemic immuneinflammation index (SII) in patients after radical operation for carcinoma of stomach in gastric cancer. J Gastrointest Oncol 2019; 10(5):965-78. doi: 10.21037/jgo.2019.05.03.

16. Zheng L, Zou K, Yang C, Chen F, Guo T, Xiong B. Inflammation-based indexes and clinicopathologic features are strong predictive values of preoperative circulating tumor cell detection in gastric cancer patients. Clin Transl Oncol 2017; 19(9):1125-32. doi: 10.1007/s12094- 017-1649-7.

17. Shi H, Jiang $Y$, Cao H, Zhu H, Chen B, Ji W. Nomogram based on systemic immune-inflammation index to predict overall survival in gastric cancer patients. Dis Markers 2018; 2018:1787424. doi: 10.1155/2018/1787424.

18. Guner A, Kim SY, Yu JE, Min IK, Roh YH, Roh C, et al. Parame- ters for predicting surgical outcomes for gastric cancer patients: Simple is better than complex. Ann Surg Oncol 2018; 25(11):3239-47. doi: 10.1245/s10434- 018-6684-2.

19. Wang K, Diao F, Ye Z, Zhang X, Zhai E, Ren H, et al. Prognostic value of systemic immune-inflammation index in patients with gastric cancer. Chin J Cancer 2017; 36(1):75. doi: 10.1186/s40880-017-0243-2.

20. Huang L, Liu S, Lei Y, Wang K, Xu M, Chen Y, et al. Systemic immune-inflammation index, thymidine phosphorylase and survival of localised gastric cancer patients after curative resection. Oncotarget 2016; 7(28):44185-93. doi: 10.18632/oncotarget.9923.

21. Wang X, Liu J, Gao A, Liu C. Effect of preoperative systemic immune-inflammation index on the prognosis of patients with gastric cancer. Acta Acad Med Weifang 2019; 41:295-8.

22. Liu X, Sun X, Liu J, Kong P, Chen S, Zhan Y, et al. Preoperative $C$-reactive protein/albumin ratio predicts prognosis of patients after curative resection for gastric cancer. Transl Oncol 2015; 8(4):339-45. doi: 10.1016/ j.tranon.2015. 06.006 .

23. Deshmukh SK, Srivastava SK, Poosarla T, Dyess DL, Holliday $\mathrm{NP}$, Singh AP, et al. Inflammation, immuno-suppressive microenvironment and breast cancer: Opportunities for cancer prevention and therapy. Ann Transl Med 2019; 7(20):593. doi: 10.21037/ atm.2019.09.68.

24. Carvalho MI, Silva-Carvalho R, Pires I, Prada J, Bianchini R, Jensen-Jarolim $\mathrm{E}$, et al. A comparative approach of tumor-associated inflammation in mammary cancer between humans and dogs. Biomed Res Int 2016; 2016: 4917387. doi: 10.1155/2016/4917387.

25. Fridman WH, Remark R, Goc J, Giraldo NA, Becht E, Hammond SA, et al. The immune microenvironment: A major player in human cancers. Int Arch Allergy Immunol 2014; 164(1):13-26. doi: 10.1159/000362332.

26. Tjomsland V, Niklasson L, Sandström P, Borch K, Druid H, Bratthäll $C$, et al. The desmoplastic stroma plays an essential role in the accumulation and modulation of infiltrated immune cells in pancreatic adenocarcinoma. Clin Dev Immunol 2011; 2011:212810. doi: 10.1155/2011/ 212810.

27. Maeda T, Hayashi T, Furukawa H, Iwasaki D, Ishikawa K, Funayama $\mathrm{E}$, et al. Immune-mediated antitumor effect of a transplanted lymph node. Int J Cancer 2018; 143(5): 1224-35. doi: 10.1002/ijc.31414.

28. Dufies M, Grytsai O, Ronco C, Camara O, Ambrosetti D, Hagege A, et al. New CXCR1/CXCR2 inhibitors represent an effective treatment for kidney or head and neck cancers sensitive or refractory to reference treatments. Theranostics 2019; 9(18):5332-46. doi: 10.7150/ thno.34681.

29. Qiao Y, Zhang C, Li A, Wang D, Luo Z, Ping Y, et al. IL6 derived from cancer-associated fibroblasts promotes chemoresistance via CXCR7 in esophageal squamous cell carcinoma. Oncogene 2018; 37(7):873-83. doi: 10.1038/onc.2017.387.

30. Mirili C, Paydas S, Kapukaya TK, Yılmaz A. Systemic immune-inflammation index predicting survival outcome in patients with classical Hodgkin lymphoma. Biomark Med 2019; 13(18):1565-75. doi: 10.2217/bmm-2019-0303.

31. Wang D, Guo D, Shi F, Zhu Y, Li A, Kong L, et al. The predic- 
tive effect of the systemic immune-inflammation index for patients with small-cell lung cancer. Future Oncol 2019; 15(29):3367-79. doi: 10.2217/fon-2019-0288.

32. Gao Y, Guo W, Cai S, Zhang F, Shao F, Zhang G, et al. Systemic immune-inflammation index (SII) is useful to predict survival outcomes in patients with surgically resected esophageal squamous cell carcinoma. J Cancer 2019; 10(14):3188-96. doi: 10.7150/jca.30281.

33. Keeley T, Costanzo-Garvey DL, Cook LM. Unmasking the many faces of tumor-associated neutrophils and macrophages: Considerations for targeting innate immune cells in cancer. Trends Cancer 2019; 5(12):789-98. doi: 10.1016/j.trecan.2019.10.013.

34. Xu SS, Xu HX, Wang WQ, Li S, Li H, Li TJ, et al. Tumor-infiltrating platelets predict postoperative recurrence and survival in resectable pancreatic neuroendocrine tumor. World J Gastroenterol 2019; 25(41):6248-57. doi: 10.3748/wjg.v25.i41.6248.

35. He Y, Wang L, Liu W, Zhong J, Bai S, Wang Z, et al. MAP3K3 expression in tumor cells and tumor-infiltrating lymphocytes is correlated with favorable patient survival in lung cancer. Sci Rep 2015; 5:11471. doi: 10.1038/ srep11471. 